\title{
Duchenne and Becker Muscular Dystrophy: Contribution of a Molecular and Immunohistochemical Analysis in Diagnosis in Morocco
}

\author{
Hanane Bellayou, ${ }^{1,2}$ Khalil Hamzi, ${ }^{1}$ Mohamed Abdou Rafai, ${ }^{3}$ Mehdi Karkouri, ${ }^{4}$ \\ Ilham Slassi, ${ }^{3}$ Houssine Azeddoug, ${ }^{2}$ and Sellama Nadifi ${ }^{1}$ \\ ${ }^{1}$ Genetic and Molecular Pathology Laboratory, Medical School, Hassan II University, 19, rue Tarik-Ibn-Ziad, BP 9154, \\ 10000 Casablanca, Morocco \\ ${ }^{2}$ UFR of Biology and Healthy, Laboratory of Biochemistry and Molecular Biology, Faculty of Sciences, Hassan II University, \\ Ain Chock, PB 5366, Maarif, 20100 Casablanca, Morocco \\ ${ }^{3}$ Neurology Department, Ibn Rochd Hospital, District Hospitals, Casablanca, Morocco \\ ${ }^{4}$ Anathomopathology Department, Ibn Rochd Hospital, District Hospitals, Casablanca, Morocco
}

Correspondence should be addressed to Sellama Nadifi, nadifi@fmpc.ac.ma

Received 10 July 2008; Revised 29 December 2008; Accepted 24 February 2009

Recommended by Kanury Rao

Duchenne muscular dystrophy (DMD) and Becker muscular dystrophy (BMD) are X-linked recessive disorders caused by mutations of the DMD gene located at Xp21. In DMD patients, dystrophin is virtually absent; whereas BMD patients have $10 \%$ to $40 \%$ of the normal amount. Deletions in the dystrophin gene represent $65 \%$ of mutations in DMD/BMD patients. To explain the contribution of immunohistochemical and genetic analysis in the diagnosis of these dystrophies, we present 10 cases of DMD/BMD with particular features. We have analyzed the patients with immunohistochemical staining and PCR multiplex to screen for exons deletions. Determination of the quantity and distribution of dystrophin by immunohistochemical staining can confirm the presence of dystrophinopathy and allows differentiation between DMD and BMD, but dystrophin staining is not always conclusive in BMD. Therefore, only identification involved mutation by genetic analysis can establish a correct diagnosis.

Copyright (C) 2009 Hanane Bellayou et al. This is an open access article distributed under the Creative Commons Attribution License, which permits unrestricted use, distribution, and reproduction in any medium, provided the original work is properly cited.

\section{Introduction}

The muscular dystrophy is a group of inherited disorders characterized by progressive muscle weakness. The best known are X-linked disorder Duchenne muscular dystrophy (DMD) [MIM 310200] and Becker muscular dystrophy (BMD) [MIM 300376]. DMD is the most common muscle disease in children, with an incidence of around 1 in 3500 live born males [1]. Affected boys are usually wheelchair bound by the age of 12 and die early in their third decade of life from respiratory or cardiac failure. BMD is a milder form of the disease with a later age of onset and a slower clinical progression.

The DMD gene [MIM 300377] is the largest human gene ever known, spanning $2.4 \mathrm{Mb}$ on Xp21 [2]. It consists of
79 exons and coding for dystrophin. 65\% of DMD/BMD cases show partial gene deletions $(60 \%)$ or duplications (5\%) of different sizes that are preferentially clustered in two major hotspots of mutation, spanning exons 3-7 and 44-55. Point mutations and small deletion/insertion account for the remaining cases (35\%) [3-5]. The difference between DMD and BMD explained by the reading frame hypothesis, mutations which disrupt the reading frame causing a premature termination and loss of dystrophin, is leading to a severe phenotype DMD. Mutations that retain the reading frame generate a shortened protein; the dystrophin may still have been limited to almost normal function, leading to a milder phenotype BMD [6, 7].

The autosomal recessive limb-girdle muscular dystrophies (LGMD) (MIM 253700; MIM 600119; MIM 604286; 
MIM 601287) are heterogeneous group of disorders resulting from mutation in any of four genes coding for the sarcoglycan proteins $\alpha-, \beta-, \gamma$-, and $\delta$-sarcoglycans. The clinical spectrum of LGMD or sarcoclycanopathies varies from Duchenne-like to late-onset mild and slowly progressive muscular dystrophy [8]. $\gamma$-sarcoglycanopathy is the most common sarcoglycanopathy in the North African population due to a founder mutation, the 521delT in the $\gamma$-sarcoglycan gene localized in 13q21 $[9,10]$.

The sarcoglcan proteins and dystrophin, are a part of the large dystrophin-associated glycoproteins (DAGs) complex, which is necessary for maintaining the link between the cytoskeleton and the extracellular matrix and also essential for preserving of the integrity of the muscle cell membrane [11].

In DMD patients, immunohistoanalysis shows total absence of dystrophin; whereas BMD patients have $10 \%$ to $40 \%$ of the normal amount or produce a partly functional dystrophin protein with an altered size [12]. For patients with sarcoglycanopathies, immunofluorescence analyses showed that absence or reduction in one of the sarcoglycan proteins has consequences on the stability of the entire sarcoglycan complex and sometimes reduction of dystrophin level as well.

In order to reach a diagnosis and provide accurate counselling for patients, and particularly the boys, affected by severe muscular dystrophy, it is necessary to identify which protein is primarily involved and thereby direct genetic analysis accordingly.

The objective of this study is to report the contribution of pathology and molecular genetics in diagnostic strategy Duchenne and Becker muscular in Morocco and in the differentiation between DMD and BMD. For that we expose only 10 cases that represent a sample of all our patients with some clinical, histopathological, and genetic features.

\section{Materials and Methods}

2.1. Patients. All patients were diagnosed in the neurological department of CHU Ibn Rochd, Casablanca. Patients showed progressive muscle weakness, calf hypertrophy, and elevated serum creatine kinase levels (4-100 times the normal value). Routine histological analysis of muscle biopsies revealed a dystrophic myopathy in all cases.

2.2. Immunostaining. Muscle biopsies were analyzed immunohistochemically using the mouse monoclonal antibody Dys-C against C-terminal domain of dystrophin and mouse monoclonal antibodies against $\alpha$ - and $\gamma$ sarcoglycan (NovoCastra, Newcastle upon Tyne, UK), with immunoperoxidase labeling $[6,13,14]$.

2.3. Mutation Analysis. PCR deletion analysis of the dystrophin gene was performed by PCR multiplex with the primer sets of Chamberlain et al. [15] and Beggs et al. [16].

The Chamberlain reaction uses primers for exons 45,48 , $19,17,51,8,12,44,4$ and the Beggs reaction primers for the muscular promoter and exons $3,43,50,13,6,47,60$, and 52. The PCR product was separated on $2 \%$ Nusieve $+1 \%$ agarose gel and detected in U.V light after ethidium bromide staining. Deletions was diagnosed when one of the bands present in the amplified control DNA was absent from the patient DNA.

Screening for del521T mutation of the $\gamma$-sarcoglycan gene was performed by PCR and directly sequenced. After amplification, $1 \mu \mathrm{L}$ samples were electrophresed using an ABI PRISM 3130xl genetic analyzer (Applied Biosystems). The data obtained were analyzed with GeneMapper analysis software V3.2 (Applied Biosystems) according to the manufacturer's recommendations.

\section{Results and Discussion}

We present an analysis of 10 boys with a muscular dystrophy; they are still ambulant with calf hypertrophy and elevated SCK activity. The patients underwent to immunohistochemistry analysis and molecular examinations (Table 1).

Contribution of the Immunohistochemical Investigation and the Molecular Survey. On the basis of expression patterns of dystrophin and $\gamma$-sarcoglycan, the patients were classified into 4 groups.

The first group, of 6 patients, showed complete absence of dystrophin and normal expression of $\alpha$ - and $\gamma$ sarcoglycans, suggesting DMD phenotype.

In the second group with 2 patients, the pattern consists of an irregular Dys-C imunoreactivity with preservation of $\alpha$ - and $\gamma$-sarcoglycans, usually determine a BMD phenotype.

In 7 patients of both groups, the deletions in dystrophin gene were revealed by multiplex PCR. Patient 1 had a maternal uncle with muscular dystrophy died after a cardiac complication; and the mothers of patients 2 and 7 have high SCK levels suggesting the conductive mother. Moreover, these 3 patients were born of a nonconsanguineous marriage, all this information leads to an X-linked transmission, which was subsequently confirmed by immunohistochemistry and genetic examinations. However, for patients 3 and 4, only no consanguinity of parents can point to a possible Xlinked transmission. Whereas, for the patients 5 and 8 , with a first degree of consanguinity and no similar cases in the family, the immunohistochemical analysis directed the molecular genetics investigation to confirm diagnosis. For the patient 6 , no deletion was found. This patient has an elder brother with the same symptoms and shows no consanguinity. Therefore, further investigations should be made to research the duplications or point mutations on the dystrophin gene, since the family inquiry and the immunohistochemical survey lead to a diagnosis of X-linked dystrophinopathy.

In the third group (patient 9), significant reduction of Dys-C, $\alpha$ - and $\gamma$-sarcoglycans immunofluorescence was seen, indicating a dystrophinopathy, but such pattern has likewise been observed in particular $\gamma$-sarcoglycanopathy. Since the consanguinity of parents and the simplicity of the technique, we searched the del521T in $\gamma$-sarcoglycan gene at first, and the mutation was not found. Subsequently, the multiplex 
TABLE 1: Clinical data, and genetic and immunohistochemical results for our patients.

\begin{tabular}{|c|c|c|c|c|c|c|c|c|}
\hline \multirow{2}{*}{$\mathrm{N}^{\circ}$} & \multirow{2}{*}{$\begin{array}{l}\text { Age } \\
\text { (years) }\end{array}$} & \multirow{2}{*}{$\begin{array}{l}\text { Age at onset } \\
\text { (years) }\end{array}$} & \multirow{2}{*}{ Consanguinity } & \multicolumn{3}{|c|}{ Immunochemistry } & \multirow{2}{*}{ Deleted exons } & \multirow{2}{*}{ Other criterion } \\
\hline & & & & Dys-C & $\alpha$-SG & $\gamma$-SG & & \\
\hline 1 & 13 & 5 & No & - & +++ & +++ & 50,51 , and 52 & $\begin{array}{l}\text { Elevated SCK activity a } \\
\text { the mother }\end{array}$ \\
\hline 2 & 9 & 3 & No & - & +++ & +++ & $\begin{array}{l}45,47,48,50 \\
51 \text {, and } 52\end{array}$ & Maternal uncle died \\
\hline 3 & 12 & 4 & No & - & +++ & +++ & 51 and 52 & $\begin{array}{l}\text { Elevated SCK activity a } \\
\text { the mother }\end{array}$ \\
\hline 4 & 16 & 5 & No & - & +++ & +++ & - & $\begin{array}{l}\text { Elder brother with the } \\
\text { same symptoms }\end{array}$ \\
\hline 5 & 11 & 3 & Yes & - & +++ & +++ & 45 and 48 & - \\
\hline 6 & 13 & 3 & No & - & +++ & +++ & 45 & - \\
\hline 7 & 12 & 5 & No & $+1-$ & +++ & +++ & $\begin{array}{l}12,13,17,19, \\
43,44,45,47 \\
\text { and } 48\end{array}$ & - \\
\hline 8 & 13 & 5 & Yes & $+1-$ & +++ & +++ & $3,4,6$, and 8 & - \\
\hline 9 & 13 & 2 & Yes & + & + & + & 48 & $\begin{array}{l}521 \mathrm{del} \mathrm{T} \text { in } \gamma \text {-SG gene } \\
\text { not found }\end{array}$ \\
\hline 10 & 19 & 11 & Yes & +++ & + & + & $\mathrm{Pm}$ & $\begin{array}{l}521 \mathrm{del} \mathrm{T} \text { in } \gamma \text {-SG gene } \\
\text { not found }\end{array}$ \\
\hline
\end{tabular}

+++: normal; +: reduced; +/-: irregular; - : absent

$\mathrm{N}^{\circ}$ : patient number; SG: sarcoglycan; Dys: dystrophin; SCK: serum creatine kinase.

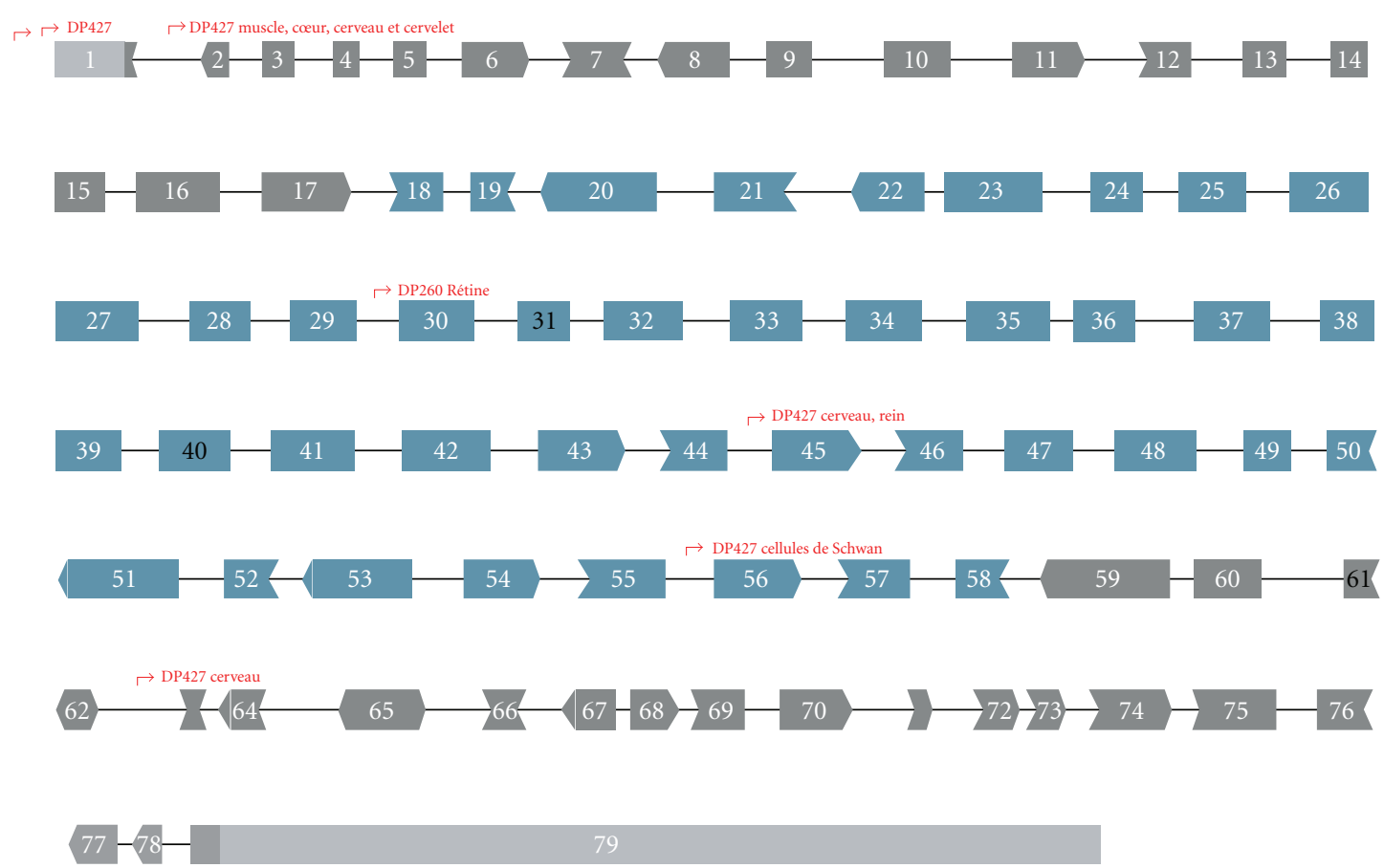

FIGURE 1: Schematic representation of the 79 exons of dystrophin gene explains the reading frame rule of Monaco et al.

PCR identified the deletion of exon 48 in the dystrophin gene.

In the latter group (patient 10), $\alpha$ - and $\gamma$-sarcoglycans were severely reduced, and staining for Dys-C were clearly detectable. This pattern related to $\gamma$-sarcoglycanopathy, in addition, the clinical information indicates the autosomal recessive trait and mild muscular dfystrophy. However; the del521T has not been found in $\gamma$-sarcoglycan gene; furthermore screening of the dystrophin gene revealed the deletion of exon 1. 
The staining pattern of patient 9 was not specific for BMD as it was also found in patients with $\alpha$ - and $\gamma$ sarcoglycanopathy, thus the immunoanalysis is unable to guide the correct molecular genetic analysis in a given case. In addition, the immunohistochemical examination with Dys$\mathrm{C}$ antibody against the C-Terminal part of the dystrophin alone can be misleading, as depicted in patient 10 . There are 2 more antibodies; one is specific to the central domain and the other to the N-Terminal part that should be used to direct the genetic analysis accordingly. The immunohistochemistry analysis is little quantitative and does not discern the truncated proteins as well [17]. Therefore, the western blot analysis, to consist on the separation of the proteins by their molecular weights, which detect the truncated proteins, may provide useful information in a given case of BMD [18].

In Duchenne and Becker muscular dystrophy, the clinical examination determines the severity of dystrophy and mode of transmission. Then, the recognition of protein deficiency is important for guiding the genetic analysis directly to the responsible gene, but in some cases, the immunohistochemical analysis can be misdiagnosed (patients 9 and 10). Therefore, only identification of mutation can conclude a correct diagnosis.

So far, we research the most frequent deletions at the hotspot region, depicting $60 \%$ of the mutations incriminated, by multiplex PCR. Henceforth, a quantitative PCR multiplex allows the simultaneous research of the deletions and duplications in all exons.

Indeed, the definition of the deletion is necessary to propose a genetic counselling and particularly prenatal diagnosis for the family. For families without deletion, we can only propose linkage analysis using polymorphic markers to female carriers.

The Correlation Genotype Phenotype DMD/BMD. The clinical discrimination between DMD and BMD is linked to the age at which the patient become weelchair dependent. BMD patients remain ambulant until the age of 16 , while DMD patients are wheelchair dependent before the age of 13 years. When patients are younger than 12 years, it is difficult to establish a diagnosis. The immunohistochemical investigation is the first tool to guide diagnosis; irregular or decreased expression of dystrophin suggests a BMD or a $\gamma$-sarcoglycanopathy if the $\gamma$-sarcoglycan was also absent (patients 7, 8, and 9). When the dystrophin was completely absent, the diagnosis was still incomplete (patients 1, 2, 3, 4, 5 , and 6).

The rule of Monaco [6] stipulates that the difference between DMD and BMD depends on the reading frame (Figure 1). If the mutation maintains an open reading frame, a shortened but functional protein and a milder phenotype (BMD) are observed. If the mutation shifts the translational reading frame of the transcripts or creates a stop codon, a severe phenotype (DMD) is observed and no protein is present.

For patients 7,8 , and 9 , the deletions maintain the reading frame as they are not very large and are located in the region coding for the central rod domain of dystrophin,

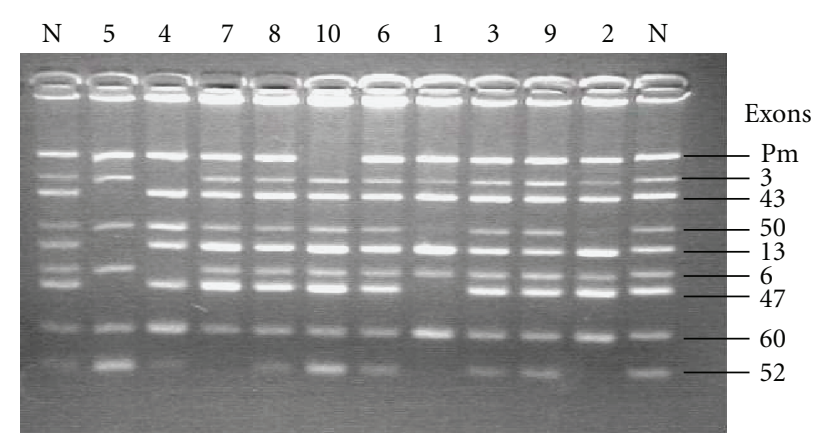

FIgURE 2: Analysis of dystrophin gene by multiplex PCR. (The primer sets of Chamberlain et al.). The top numbers correspond to the codes of patients. The numbers at the right indicate the amplified exons. N: Normal control with all exons. Patient 2 with deletion of exons 50 and 52. Patient 1 with deletion of exons 50, 47, and 52. Patient 10 with deletion of promoter Pm. Patient 7 with deletion of exon 52. Patient 4 with deletion of exons 3 and 6. Patient 5 with deletion of exons 43,13 , and 47.

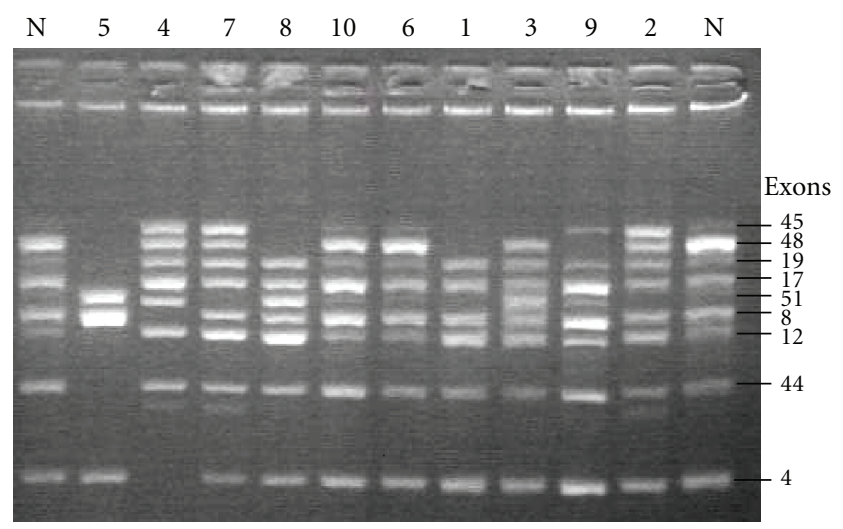

FIGURE 3: Analysis of dystrophin gene by multiplex PCR. (The primer sets of Beggs et al.). The top numbers correspond to the codes of patients. The numbers at the right indicate the amplified exons. N: Normal control with all exons. Patient 2 with deletion of exon 51. Patient 9 with deletion of exon 48. Patient 3 with deletion of exon 45. Patient 1 with deletion of exons 45, 48, and 51. Patient 8 with deletion of exons 45 and 48 . Patient 7 with deletion of exon 51. Patient 4 with deletion of exons 8 and 4 . Patient 5 with deletion of exons $12,19,44,45,47$, and 4 .

that confirms the results of immunoanalysis of a BMD. For patients 1,2 , and 5 , deletions disrupt the reading frame conducting a DMD phenotype. For patient 3, deletion of only exon 45 was found, with the presence of exons 44 and 47 , in this case we cannot conclude. Accordingly, the deletion affects or not exon 46; if it is deleted the reading frame will be restoring and the BMD phenotype will appear (Figures 1 and 2).

For 4 and 10, the age of the patients indicates a BMD diagnosis, since the patients are still ambulant. The deletion of exons 3, 4, 6, and 8 was found in patient 4 (Figures 1 and 2). The reading frame is maintained, but this region presents one of the exceptions to the rule of Monaco [19]. On the region of exon 3 in exon 7 , the deletion can produce both 
phenotypes DMD and BMD. This patient of 16 years is still ambulant suggesting a BMD diagnosis. For patient 10, the deletion of exon 1 promoter was revealed. The expression of dystrophin was preserved, that could be explained by the presence of several more promoters in the dystrophin gene.

\section{Conclusion}

The protein-based strategy, followed by molecular analysis, is probably the most logical and efficient way to approach diagnosis of Duchenne and Becker muscular dystrophy in Morocco where sarcoglycanopathy DMD-like is the most frequent muscular dystrophy.

However, complete immunohistochemistry analysis with all available dystrophin antibodies, therefore, is a useful tool to guide the molecular genetic investigations that are necessary to arrive at the correct genetic diagnosis in a given case.

\section{Acknowledgments}

The authors express their thanks for the patient and families for their participation in the study. They also thank Dr F. Leturcq and Mr S. Llense from Institute Cochin for their technical advice and Pr. A. Mazabraud for his contributions to this study.

\section{References}

[1] A. E. H. Emery, "Population frequencies of inherited neuromuscular diseases - a world survey," Neuromuscular Disorders, vol. 1, no. 1, pp. 19-29, 1991.

[2] M. Koening, E. P. Hoffman, C. J. Bertelson, A. P. Monaco, C. Feener, and L. M. Kunkel, "Complete cloning of the Duchenne muscular dystrophy (DMD) cDNA and preliminary genomic organization of the DMD gene in normal and affected individuals," Cell, vol. 50, no. 3, pp. 509-517, 1987.

[3] S. M. Forrest, G. S. Cross, T. Flint, A. Speer, K. J. H. Robson, and K. E. Davies, "Further studies of gene deletions that cause Duchenne and Becker muscular dystrophies," Genomics, vol. 2, no. 2, pp. 109-114, 1988.

[4] J. T. Den Dunnen, P. M. Grootscholten, E. Bakker, et al., "Topography of the Duchenne muscular dystrophy (DMD) gene: FIGE and cDNA analysis of 194 cases reveals 115 deletions and 13 duplications," The American Journal of Human Genetics, vol. 45, no. 6, pp. 835-847, 1989.

[5] X. Hu, A. H. Burghes, P. N. Ray, M. W. Thompson, E. G. Murphy, and R. G. Worton, "Partial gene duplication in Duchenne and Becker muscular dystrophies," Journal of Medical Genetics, vol. 25, no. 6, pp. 369-376, 1988.

[6] A. P. Monaco, C. J. Bertelson, S. Liechti-Gallati, H. Moser, and L. M. Kunkel, "An explanation for the phenotypic differences between patients bearing partial deletions of the DMD locus," Genomics, vol. 2, no. 1, pp. 90-95, 1988.

[7] M. Koening, A. H. Beggs, M. Moyer, et al., "The molecular basis for Duchenne versus becker muscular dystrophy: correlation of severity with type of deletion," The American Journal of Human Genetics, vol. 45, no. 4, pp. 498-506, 1989.
[8] K. M. Bushby, "The limb-girdle muscular dystrophies: diagnostic guidelines," European Journal of Paediatric Neurology, vol. 3, no. 2, pp. 53-58, 1999.

[9] K. Ben Othmane, M. C. Speer, J. Stauffer, et al., "Evidence for linkage disequilibrium in chromosome 13-linked Duchennelike muscular dystrophy (LGMD2C)," The American Journal of Human Genetics, vol. 57, no. 3, pp. 732-734, 1995.

[10] M. Kefi, R. Amouri, A. Driss, et al., "Phenotype and sarcoglycan expression in Tunisian LGMD 2C patients sharing the same del521-T mutation," Neuromuscular Disorders, vol. 13, no. 10, pp. 779-787, 2003.

[11] M. Yoshida and E. Ozawa, "Glycoprotein complex anchoring dystrophin to sarcolemma," Journal of Biochemistry, vol. 108, no. 5, pp. 748-752, 1990.

[12] E. P. Hoffman, K. H. Fischbeck, R. H. Brown, et al., "Characterization of dystrophin in muscle-biopsy specimens from patients with Duchenne's or Becker's muscular dystrophy," The New England Journal of Medicine, vol. 318, no. 21, pp. 1363-1368, 1988.

[13] E. P. Hoffman, R. H. Brown Jr., and L. M. Kunkel, "Dystrophin: the protein product of the Duchenne muscular dystrophy locus," Cell, vol. 51, no. 6, pp. 919-928, 1987.

[14] A. Bonnermann and L. V. Anderson, "Diagnostic protein expression in human muscle biopsies," Brain Pathology, vol. 10, no. 2, pp. 193-214, 2000.

[15] J. S. Chamberlain, R. A. Gibbs, J. E. Ranier, P. N. Nguyen, and C. T. Caskey, "Deletion screening of the Duchenne muscular dystrophy locus viamultiplex DNA amplification," Nucleic Acids Research, vol. 16, no. 23, pp. 11141-11156, 1988.

[16] A. H. Beggs, M. Koenig, F. M. Boyce, and L. M. Kunkel, "Detection of $98 \%$ of DMD/BMD gene deletions by polymerase chain reaction," Human Genetics, vol. 86, no. 1, pp. 45-48, 1990.

[17] C. A. Sewry, "Immunocytochemical analysis of human muscular dystrophy," Microscopy Research and Technique, vol. 48, no. 3-4, pp. 142-154, 2000.

[18] L. V. B. Anderson and K. Davison, "Multiplex Western blotting system for the analysis of muscular dystrophy proteins," The American Journal of Pathology, vol. 154, no. 4, pp. 1017-1022, 1999.

[19] A. V. Winnard, C. J. Klein, D. D. Coovert, et al., "Characterization of translational frame exception patients in Duchenne/Becker muscular dystrophy," Human Molecular Genetics, vol. 2, no. 6, pp. 737-744, 1993. 

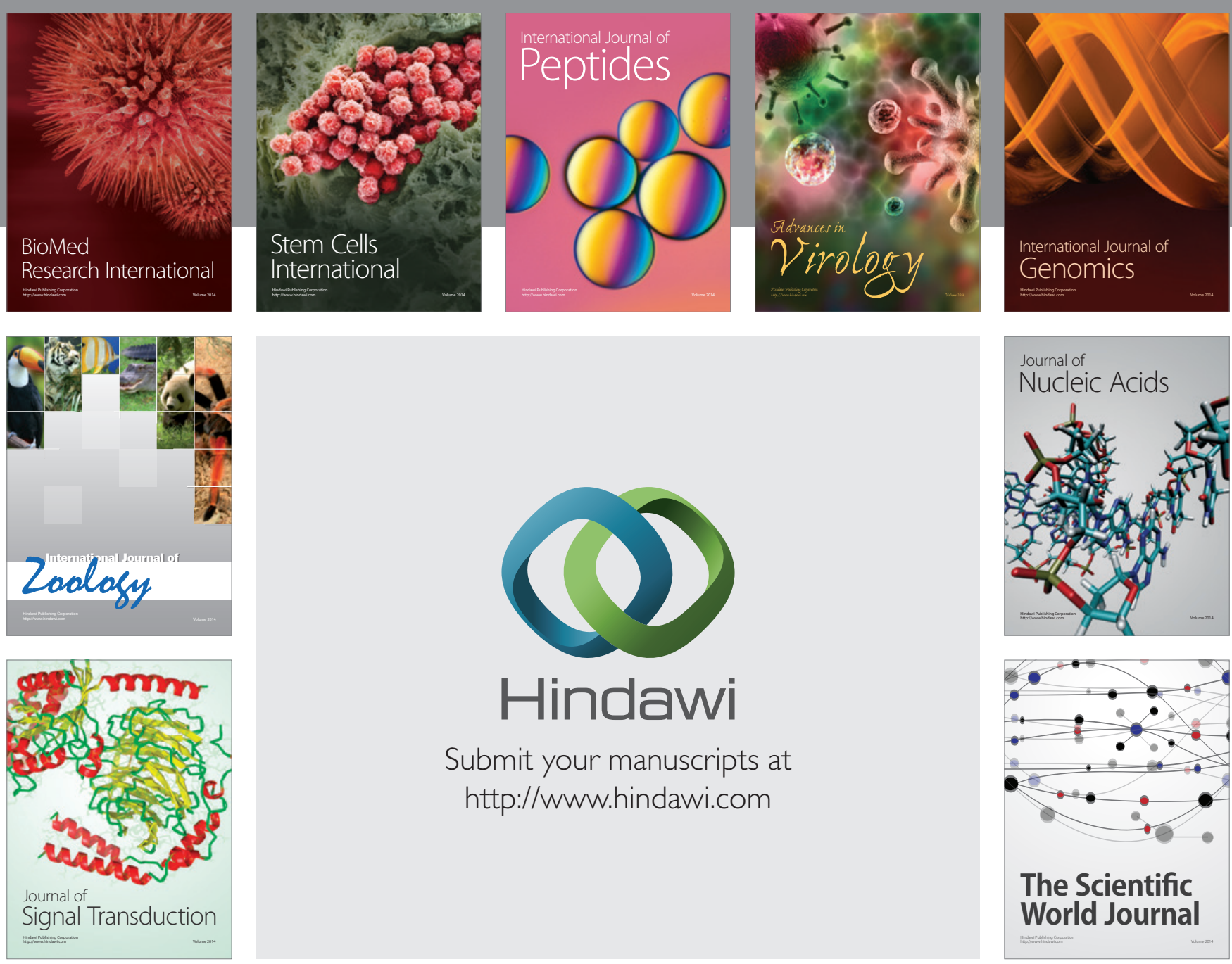

Submit your manuscripts at

http://www.hindawi.com
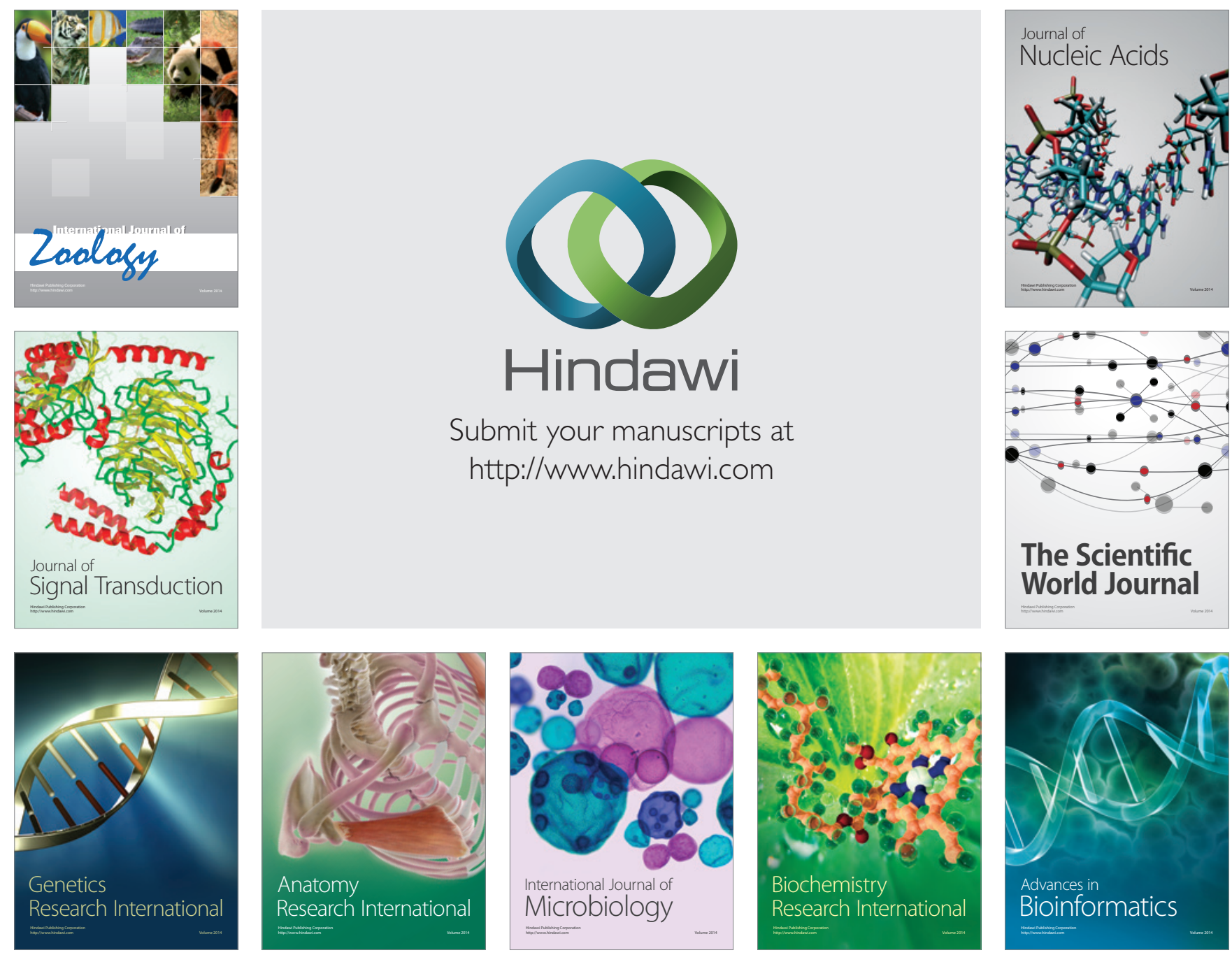

The Scientific World Journal
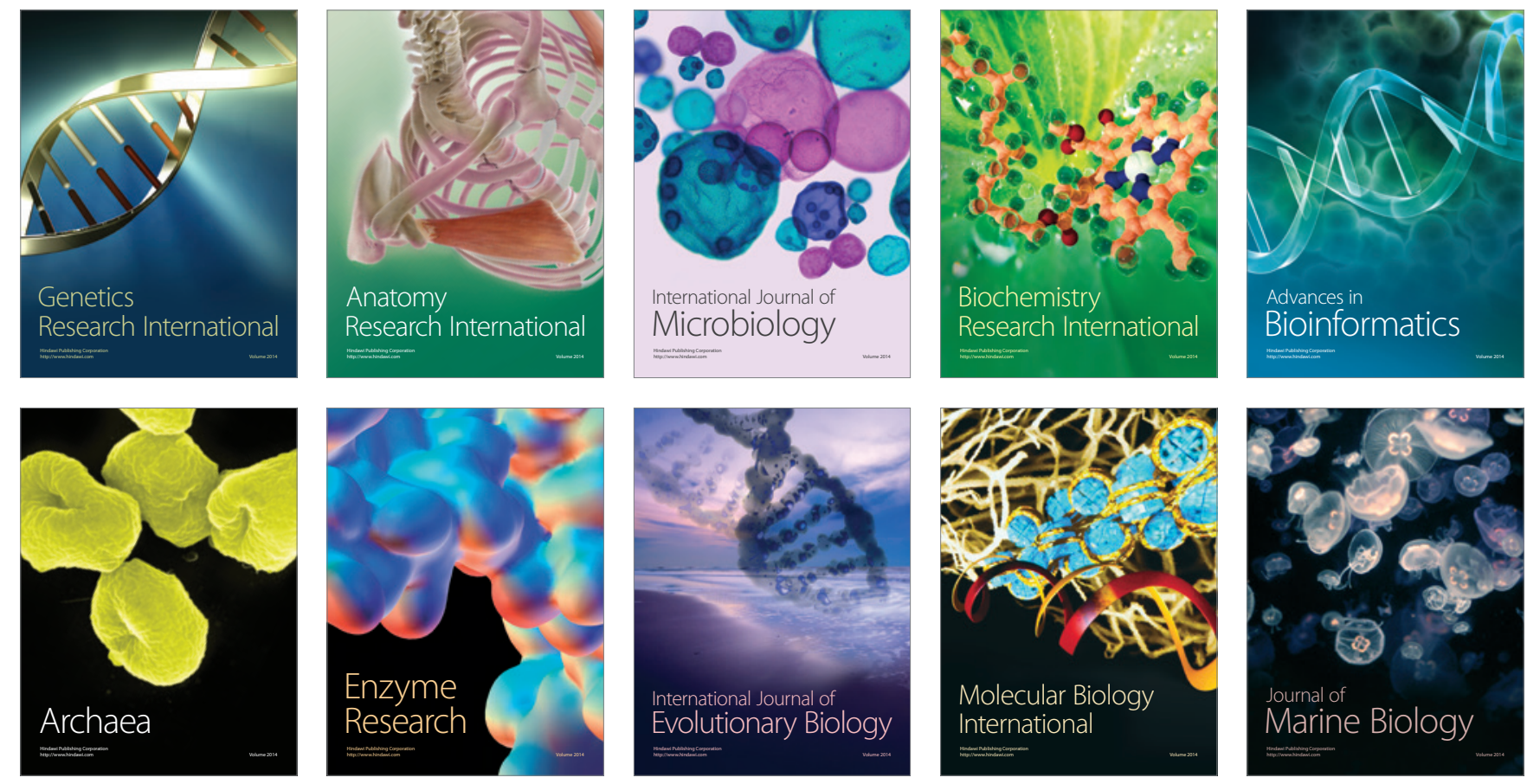